\title{
A REMARK ON THE GROMOV CONVERGENCE THEOREM
}

\author{
YUKIO OTSU
}

(Communicated by Jonathan M. Rosenberg)

\begin{abstract}
In [3] M. Gromov introduced the concept of convergence of Riemannian manifolds and he proved the convergence theorem. Since that time the theorem has been developed in detail (see [5], [7], [2]), and we know that it contains some interesting applications. Nevertheless there seems to be an inadequate way of applying the convergence theorem. The purpose of this paper is to present an example which shows that it is not correct.
\end{abstract}

\section{INTRODUCTION}

First of all we recall the convergence theorem. Let $M$ be an $n$-dimensional compact Riemannian manifold. We use the notation $K_{M}$ for the sectional curvature of $M, \operatorname{vol}(M)$ for the volume and $\operatorname{diam}(M)$ for the diameter. Let $\mathscr{M}=\mathscr{M}(n, \Delta, D, v)$ denote the class of all $n$-dimensional Riemannian manifolds $M$ satisfying $\left|K_{M}\right| \leq \Delta, \operatorname{diam}(M) \leq D, \operatorname{vol}(M) \geq v>0$.

Theorem 1.1 (Katsuda [5], Peters [7]). The Hausdorff topology coincides with the Lipschitz topology on $\mathscr{M}$.

Furthermore, we have:

Theorem 1.2 (Peters [7], Greene-Wu [2]). Given $0<\alpha<1$, let $\left\{\left(M_{i} g_{i}\right)\right\}_{i=1,2, \ldots}$ be a sequence of Riemannian manifolds in $\mathscr{M}$. Then there exist a $C^{1, \alpha}$ Riemannian manifold $\left(M, g_{\infty}\right)$ and a subsequence $\left\{\left(M_{i_{j}}, g_{i_{j}}\right)\right\}_{j=1,2, \ldots}$ which converges to $\left(M, g_{\infty}\right)$ with respect to Lipschitz topology.

For simplicity we denote the subsequence $\left\{i_{j}\right\}_{j=1,2, \ldots}$ by $\{j\}$. In the above case, by definition, there exist diffeomorphisms $f_{j}: M \rightarrow M_{j}$ such that $\left\{g_{j}^{\prime}:=\right.$ $\left.f_{j}^{*} g_{j}\right\}$ converges to $g_{\infty}$ on $M$. Let $p \in M$. The metric $g_{j}^{\prime}$ induces the exponential map $\exp _{p}^{g_{j}^{\prime}}: T_{p} M \rightarrow M$. By Rauch's comparison theorem $\exp _{p}^{g_{\infty}}$ is Lipschitz continuous. Hence $\exp _{p}^{g_{\infty}}$ is differentiable almost everywhere. Thus it is reasonable to expect the following (see [1], [4, 5.15]) proposition.

\footnotetext{
Received by the editors June 2, 1988, and, in revised form August 15, 1988 and January 27, 1989.

1980 Mathematics Subject Classification (1985 Revision). Primary 53C20; Secondary 51H25.

Key words and phrases. Convergence theorem, Pinching problem.
} 
Proposition 1.3. At any point in the tangent space $T_{p} M$ where $d \exp _{p}^{g_{\infty}}$ makes sense

$$
\lim _{J \rightarrow \infty} d \exp _{p}^{g_{j}^{\prime}}=d \exp _{p}^{g_{\infty}} .
$$

However, Proposition 1.3 is false. The proof of a diameter pinching theorem ([1]) contains the same mistake (for another proof of the theorem, see [5], also [8]). In this paper we present a simple counterexample to the proposition.

\section{A Counterexample}

Let $M_{\infty}=\left(M, g_{\infty}\right)$ be a 2-dimensional compact Riemannian manifold with injectivity radius at least 2 and let $p$ be a point of $M$. We construct a sequence of compact Riemannian manifolds $M_{i}=\left(M, g_{i}\right)$ from $\left(M, g_{\infty}\right)$ as follows. Let $\varphi: B_{p}(2) \backslash\{p\} \rightarrow(0,2) \times S^{1}$ be the polar coordinate system centered at $p$. Assume that $g_{\infty}$ is written as

$$
\left(\varphi^{-1}\right)^{*} g_{\infty}=d r^{2}+G_{\infty}^{2}(r) d \theta^{2}
$$

at $(r, \theta) \in(0,2) \times S^{1}$ for some $C^{\infty}$-function $G_{\infty}$. Let $\lambda: \mathbf{R} \rightarrow[0,1 / 2]$ denote a nonnegative $C^{\infty}$-function such that

$$
\begin{array}{ll}
\lambda(r)=0 & \text { if } r \leq \frac{1}{4}, 1 \leq r, \\
\lambda(r)=1 / 2 & \text { if } \frac{1}{2} \leq r \leq \frac{3}{4} .
\end{array}
$$

For any integer $i$ the metric $g_{i}$ on $B_{p}(2) \backslash\{p\}$ is defined to be

$$
\left(\varphi^{-1}\right)^{*} g_{i}=d r^{2}+G_{i}^{2}(r, \theta) d \theta^{2},
$$

where $G_{i}(r, \theta):=G_{\infty}(r)(1+\lambda(r) \cos i \theta)$. Since $G_{i}=G_{\infty}$ for $r \leq 1 / 4$ and $1 \leq r$, we can extend $g_{i}$ on $M$ so that $g_{i}=g_{\infty}$ on $B_{p}(1 / 4)$ and $M \backslash B_{p}(1)$.

We show that the sequence $\left\{M_{i}\right\}_{i=1,2, \ldots}$ obtained as above is in $\mathscr{M}(2, \Delta, D, v)$ for some constants $\Delta, D, v$. It is sufficient to consider $g_{i} \mid B_{p}(1)$. The sectional curvature $K_{i}$ of $g_{i} \mid B_{p}(1)$ is

$$
K_{i}=-\frac{\left(G_{i}\right)_{r r}}{G_{i}}=-\frac{\left(G_{\infty}\right)_{r r}}{G_{\infty}}-\frac{2\left(G_{\infty}\right)_{r} \lambda^{\prime}+G_{\infty} \lambda^{\prime \prime}}{G_{\infty}} \cdot \frac{\cos i \theta}{1+\lambda \cos i \theta} .
$$

Thus $K_{i}$ is uniformly bounded for $i$. The diameter of $B_{p}(1)$ with respect to $g_{i}$ is at most 2. And the volume of $B_{p}(1)$ with respect to $g_{i}$ is that of $B_{p}(1)$ with respect to $g_{\infty}$.

Claim 2.1. $d_{L}\left(M_{i}, M_{\infty}\right) \rightarrow 0$ as $i \rightarrow \infty$.

Proof. To estimate the distance between $M_{i}$ and $M_{\infty}$ we define the diffeomorphism $F_{i}: M_{i} \rightarrow M_{\infty}$ by

$$
F_{i} \mid\left(M \backslash B_{p}(1)\right)=i d_{M \backslash B_{p}(1)}
$$

and

$$
\varphi \circ\left(F_{i} \mid B_{p}(1)\right) \circ \varphi^{-1}(r, \theta)=\left(r, \theta+\frac{\lambda(r)}{i} \sin i \theta\right)
$$


Let $q$ be a point in $B_{p}(1)$ and $u$ be a unit tangent vector at $q$, then

$$
d \varphi_{q} u=\cos \phi \frac{\partial}{\partial r}+\frac{\sin \phi}{G_{i}(r)} \frac{\partial}{\partial \theta}
$$

for some $\phi$, where $\varphi(q)=(r, \theta)$. Then

$$
g_{\infty}\left(d F_{i} u, d F_{i} u\right)=\cos ^{2} \phi+G_{\infty}^{2} \cdot\left(\frac{\lambda^{\prime}}{i} \sin i \theta \cos \phi+\frac{\sin \phi}{G_{\infty}}\right)^{2} .
$$

Therefore there exists a constant $c \geq 0$ which is independent of $i$ such that for any $u$ in the unit tangent bundle of $M_{i}$,

$$
1-\frac{c}{i} \leq\left|d F_{i} u\right| \leq 1+\frac{c}{i} \text {. }
$$

Similarly, we find a constant $c^{\prime} \geq 0$ which is independent of $i$ such that for any $w$ in the unit tangent bundle of $M_{\infty}$,

$$
1-\frac{c^{\prime}}{i} \leq\left|d F_{i}^{-1} w\right| \leq 1+\frac{c^{\prime}}{i}
$$

This completes the proof.

Let $g_{i}^{\prime}:=\left(F_{i}^{-1}\right)^{*} g_{i}$. We can easily show that $\left\{d \exp _{p}^{g_{i}^{\prime}}\right\}$ does not converge for almost all $u \in T_{p} M$ with $1 / 4 \leq|u| \leq 1$ : Since $F_{i}(p)=p$ and $d F_{i}: T_{p} M_{i} \rightarrow$ $T_{p} M_{\infty}$ is an isometry, $T_{p} M_{i}$ and $T_{p} M_{\infty}$ are naturally identified as metric vector spaces. Since $\partial / \partial r$ and $1 / G_{i} \partial / \partial \theta$ make an orthonormal base,

$$
\begin{aligned}
\operatorname{det} d\left(\exp _{p}^{g_{i}^{\prime}}\right)_{u} & =\operatorname{det} d F_{i} \circ d\left(\exp _{p}^{g_{i}}\right)_{u} \\
& =(1+\lambda \cos i \theta) \times \frac{G_{i}}{r} \\
& =(1+\lambda \cos i \theta)^{2} \times \frac{G_{\infty}}{r} .
\end{aligned}
$$

Similarly

$$
\operatorname{det} d\left(\exp _{p}^{g_{\infty}}\right)_{u}=\frac{G_{\infty}}{r} .
$$

Hence $\left\{\operatorname{det} d\left(\exp _{p}^{g_{i}^{\prime}}\right)_{u}\right\}$ does not converge to $\operatorname{det} d\left(\exp _{p}^{g_{\infty}}\right)_{u}$ for almost all $u \in$ $T_{p} M$ with $1 / 4 \leq|u| \leq 1$.

Let $\left\{f_{i}\right\}$ be a sequence of diffeomorphisms $f_{i}: M_{\infty} \rightarrow M_{i}, i=1,2, \ldots$. We say that $\left\{d \exp ^{i}\right\}$ converges to $d\left(\exp _{p}^{g_{\infty}}\right)$ at $p$ with respect to $\left\{f_{i}\right\}$ if $\left\{d\left(\exp _{p}^{g_{j}^{\prime}}\right)_{u}: T_{p} M \rightarrow T M\right\}$ converges to $d\left(\exp _{p}^{g_{\infty}}\right)_{u}$ for almost all $u \in T_{p} M$ for some choice of subsequence $\left\{f_{j}\right\}$ such that $\left\{g_{j}^{\prime}:=f_{j}^{*} g_{j}\right\}$ is convergent. We proved that $\left\{d \exp ^{i}\right\}$ does not converge to $d\left(\exp _{p}^{g_{\infty}}\right)$ with respect to $\left\{F_{i}^{-1}\right\}$. However $\left\{F_{i}^{-1}\right\}$ is not $C^{2, k}$-bounded on $M_{\infty}$. We shall prove in the following that there exists a sequence of $C^{2, \alpha}$-bounded diffeomorphisms which is constructed from $\left\{F_{i}^{-1}\right\}$ by the center of mass technique as in the proof of Theorem 1.2 and with respect to which $\left\{d \exp ^{i}\right\}$ does not converge to $d\left(\exp _{p}^{g_{\infty}}\right)$. 
Assume that $0<\delta \ll R \ll 1$. If $\left\{p_{\alpha}\right\}$ is a $\delta$-maximal system on $M_{\infty}$, then $\left\{F_{i}^{-1}\left(p_{\alpha}\right)\right\}$ is an almost $\delta$-net on $M_{i}$. A family of local embeddings $\phi_{\alpha}^{i}: H\left(p_{\alpha} ; R\right) \rightarrow M_{i}$ is constructed by using harmonic $R$-balls $H\left(p_{\alpha} ; R\right)$. In our case, if $p_{\alpha} \in B(p ; 1 / 4-R)$, we may consider $\phi_{\alpha}^{i}$ to be the identity on $H\left(p_{\alpha} ; R\right)$. Hence the maps $\left\{f_{i}\right\}$ constructed from $\left\{\phi_{\alpha}^{i}\right\}$ by the center of mass have the property that $f_{i}$ is identical on a neighborhood of $p$. By taking a subsequence $\left\{f_{j}\right\}$ we may assume $\left\{f_{j}\right\}$ converges to $i d_{M}$ with respect to the $C^{2, \alpha}$-norm on $M$ and $\left\{g_{j}^{\prime}:=f_{j}^{*} g_{j}\right\}$ converges to $g_{\infty}$ with respect to the $C^{1, \alpha}$ norm. Since

$$
\operatorname{det} d f_{j} \circ d\left(\exp _{p}^{g_{j}^{\prime}}\right)_{u}=\operatorname{det} d\left(\exp _{p}^{g_{j}}\right)_{u},
$$

if we suppose that $\lim _{j \rightarrow \infty} d\left(\exp _{p}^{g_{j}^{\prime}}\right)_{u}=d\left(\exp _{p}^{g_{\infty}}\right)_{u}$, then

$$
\lim _{j \rightarrow \infty} \operatorname{det} d\left(\exp _{p}^{g_{j}}\right)_{u}=\operatorname{det} d\left(\exp _{p}^{g_{\infty}}\right)_{u} \text {. }
$$

However this is impossible for almost all $u \in T_{p} M$ with $1 / 4<|u|<1$ as above.

Remark 2.2. If we take the unit sphere $S^{2}(1)$ as $M_{\infty}$ and change $\lambda$ properly, we have an example such that every $M_{i}$ has positive sectional curvature.

\section{ACKNOWLEDGMENT}

The author would like to thank Professor Katsuhiro Shiohama for his advice and encouragement. He is also indebted to Takao Yamaguchi, Kenji Fukaya, Atsuchi Katsuda, Koji Cho, Jin-ichi Itoh and Tadashi Yamaguchi.

\section{REFERENCES}

1. D. Brittain, A diameter pinching theorem for positive Ricci curvature, preprint.

2. R. Greene and H. Wu, Lipschitz convergence of Riemannian manifolds, Pacific J. Math. 131 (1988) 119-141.

3. M. Gromov, Structures métriques pour les variétés riemanniennes, Réd. par J. Lafontaine et P. Pansu, Paris, 1981.

4. K. Grove, Metric differential geometry, differential geometry, Lecture Notes in Math., Vol. 1263, 171-227, Springer-Verlag, 1985.

5. A. Katsuda, Gromov's convergence theorem and its application, Nagoya Math. J. 100 (1985), 11-48.

6. J. Kazdan, An isoperimetric inequality and Wiedersehen manifolds, in Seminar on Differential Geometry, Ann. of Math. Studies 102, 143-157, Princeton University Press, 1982.

7. S. Peters, Convergence of Riemannian manifolds, Compositio Math. 62 (1987) 3-16.

8. T. Yamaguchi, Lipschitz convergence of manifolds of positive Ricci curvature with large volume, (to appear in Math. Ann.)

Kyushu University, Department of Mathematics, Faculty of Science, Fukuoka 812, JAPAN 